\title{
AN UNIVALENT CONDITION FOR A FAMILY OF INTEGRAL OPERATORS
}

\author{
JIN-LIN LIU
}

Abstract. The object of the present paper is to derive an univalent condition for a family of integral operators.

\section{Introduction}

Let $A$ be the class of functions $f$ of the form:

$$
f(z)=z+\sum_{k=2}^{\infty} a_{k} z^{k}
$$

which are analytic in the open unit disk

$$
U=\{z: z \in C \text { and }|z|<1\}
$$

We denote by $S$ the subclass of $A$ consisting of functions which are also univalent in $U$. For some recent investigations of various interesting subclasses of the univalent function class $S$, see the works by (for example) Altintas et al. [1], Gao et al. [5], and Owa et al. [6].

The univalent conditions involving the integral operators have been studied by several authors (see, e.g., [2, 3, 4, 7, 8]). In [7] Pescar obtained the following:

Theorem 1.(see [7]). Let

$$
\alpha \in C \quad(\operatorname{Re} \alpha>0)
$$

and

$$
c \in C \quad(|c| \leq 1 ; c \neq-1) .
$$

Suppose also that the function $f(z)$ given by (1.1) is analytic in $U$. If

$$
\left.|c| z\right|^{2 \alpha}+\left(1-|z|^{2 \alpha}\right) \frac{z f^{\prime \prime}(z)}{\alpha f^{\prime}(z)} \mid \leq 1 \quad(z \in U)
$$

2010 Mathematics Subject Classification. 30C45.

Key words and phrases. Analytic function, integral operator, univalent function. 
then the function $F_{\alpha}(z)$ defined by

$$
F_{\alpha}(z)=\left(\alpha \int_{0}^{z} t^{\alpha-1} f^{\prime}(t) d t\right)^{\frac{1}{\alpha}}=z+\cdots
$$

is analytic and univalent in $U$.

Very recently, another univalent condition associated with the general family of integral operators was given by Breaz et al. [4] as follows.

Theorem 2. (see [4]) Let $M \geq 1$ and suppose that each of the functions $g_{j} \in A(j=1,2, \cdots, n)$ satisfies the inequality

$$
\left|\frac{z^{2} g_{j}^{\prime}(z)}{\left[g_{j}(z)\right]^{2}}-1\right| \leq 1 \quad(z \in U)
$$

Also let

$$
\alpha \in R \quad\left(\alpha \in\left[1, \frac{(2 M+1) n}{(2 M+1) n-1}\right]\right) \text { and } c \in C \text {. }
$$

If

$$
|c| \leq 1+\left(\frac{1-\alpha}{\alpha}\right)(2 M+1) n
$$

and

$$
\left|g_{j}(z)\right| \leq M \quad(z \in U ; j=1,2, \cdots, n),
$$

then the function $G_{n, \alpha}(z)$ defined by

$$
G_{n, \alpha}(z)=\left([n(\alpha-1)+1] \int_{0}^{z}\left[g_{1}(t)\right]^{\alpha-1} \cdots\left[g_{n}(t)\right]^{\alpha-1} d t\right)^{\frac{1}{n(\alpha-1)+1}} \quad\left(g_{1}, \cdots, g_{n} \in A\right)
$$

is in the univalent function class $S$.

In this note we propose to investigate further univalent conditions involving the general family of integral operators defined by (1.5).

\section{The main univalent condition}

Theorem 3. Let

$$
\alpha \in C \quad(\operatorname{Re} \alpha>0)
$$

and

$$
c \in C \quad(|c|<1) .
$$

Suppose that each of the functions $g_{j} \in A(j=1,2, \cdots, n)$ satisfies the condition

$$
\left|\frac{z g_{j}^{\prime}(z)}{g_{j}(z)}-1\right| \leq \frac{|\alpha|}{n(1+|\alpha|)}(1-|c|)
$$


then the function $G_{n, \alpha}(z)$ defined by (1.5) is in the univalent function class $S$.

Proof. From (1.6) and the hypothesis of the theorem, one can see that

$$
\left|\frac{z g_{j}^{\prime}(z)}{g_{j}(z)}-1\right| \leq 1 \quad(z \in U ; j=1,2, \cdots, n),
$$

which indicates that each function $g_{j}(j=1,2, \cdots, n)$ is an univalent starlike function. So the function $G_{n, \alpha}(z)$ in (1.5) is well defined.

Put

$$
f(z)=\int_{0}^{z} \prod_{j=1}^{n}\left(\frac{g_{j}(t)}{t}\right)^{\alpha-1} d t .
$$

Then it follows from (1.7) that

$$
f^{\prime}(z)=\prod_{j=1}^{n}\left(\frac{g_{j}(z)}{z}\right)^{\alpha-1}
$$

and

$$
f^{\prime \prime}(z)=(\alpha-1) \sum_{j=1}^{n}\left(\left(\frac{g_{j}(z)}{z}\right)^{\alpha-2}\left(\frac{z g_{j}^{\prime}(z)-g_{j}(z)}{z^{2}}\right) \cdot \prod_{k=1(k \neq j)}^{n}\left(\frac{g_{k}(z)}{z}\right)^{\alpha-1}\right) .
$$

We thus find from (1.8) and (1.9) that

$$
\frac{z f^{\prime \prime}(z)}{f^{\prime}(z)}=(\alpha-1) \sum_{j=1}^{n}\left(\frac{z g_{j}^{\prime}(z)}{g_{j}(z)}-1\right),
$$

which readily shows that

$$
\begin{aligned}
\left.|c| z\right|^{2 \alpha}+\left(1-|z|^{2 \alpha}\right) \frac{z f^{\prime \prime}(z)}{\alpha f^{\prime}(z)} \mid & =\left.|c| z\right|^{2 \alpha}+\left(1-|z|^{2 \alpha}\right) \frac{\alpha-1}{\alpha} \sum_{j=1}^{n}\left(\frac{z g_{j}^{\prime}(z)}{g_{j}(z)}-1\right) \mid \\
& \leq|c|+\frac{1+|\alpha|}{|\alpha|} \sum_{j=1}^{n}\left|\frac{z g_{j}^{\prime}(z)}{g_{j}(z)}-1\right| .
\end{aligned}
$$

Now by using the hypothesis (1.6), we obtain

$$
\left.|c| z\right|^{2 \alpha}+\left(1-|z|^{2 \alpha}\right) \frac{z f^{\prime \prime}(z)}{\alpha f^{\prime}(z)} \mid \leq 1 \quad(z \in U) .
$$

Finally, by applying Theorem 1, we conclude that the function $G_{n, \alpha}(z)$ defined by (1.5) is in the univalent function class $S$. This evidently completes the proof of the theorem.

Setting $c=0$ in Theorem 3, we immediately arrive at the following application of Theorem 3.

Corollary 1. Let $\alpha \in C(\operatorname{Re} \alpha>0)$ and each of the functions $g_{j} \in A(j=1,2, \cdots, n)$ satisfy the condition

$$
\left|\frac{z g_{j}^{\prime}(z)}{g_{j}(z)}-1\right| \leq \frac{|\alpha|}{n(1+|\alpha|)},
$$

then the function $G_{n, \alpha}(z)$ defined by (1.5) is in the univalent function class $S$. 


\section{References}

[1] O. Altintas, H. Irmak, S. Owa and H. M. Srivastava, Coefficient bounds for some families of starlike and convex functions of complex order, Appl. Math. Lett., 20(2007), 1218-1222.

[2] D. Breaz, Integral Operators on Spaces of Univalent Functions, Publishing House of the Romanian Academy of Sciences, Bucharest, in Romanian, 2004.

[3] D. Breaz and N. Breaz, Univalence of an integral operator, Mathematica(Cluj) 47(70) (2005), 35-38.

[4] D. Breaz, N. Breaz and H. M. Srivastava, An extension of the univalent condition for a family of integral operators, Appl. Math. Lett., 22(2009), 41-44.

[5] C.-Y. Gao, S.-M. Yuan and H. M. Srivastava, Some functional inequalities and inclusion relationships associated with certain families of integral operators, Comput. Math. Appl., 49(2005), 1787-1795.

[6] S. Owa, M. Nunokawa, H. Saitoh and H. M. Srivastava, Close-to-convexity of certain analytic functions, Appl. Math. Lett., 15(2002), 63-69.

[7] V. Pescar, A new generalization of Ahlfors's and Becker's criterion of univalence, Bull. Malaysian Math. Soc. (Ser.2) 19(1996), 53-54.

[8] V. Pescar, On the univalence of some integral operators, J. Indian Acad. Math., 27(2005), 239-243.

Department of Mathematics, Yangzhou University, Yangzhou, 225002, China.

E-mail: jlliu@yzu.edu.cn 